\title{
Requests for euthanasia: origin of suffering in ALS, heart failure, and cancer patients
}

\author{
Maud Maessen • Jan H. Veldink • \\ Leonard H. van den Berg • Henrike J. Schouten • \\ Gerrit van der Wal · Bregje D. Onwuteaka-Philipsen
}

Received: 25 August 2009/Revised: 6 December 2009/Accepted: 15 January 2010/Published online: 11 February 2010

(c) Springer-Verlag 2010

\begin{abstract}
In The Netherlands, relatively more patients (20\%) with amyotrophic lateral sclerosis (ALS) die due to euthanasia or physician-assisted suicide (EAS) compared with patients with cancer $(5 \%)$ or heart failure $(0.5 \%)$. We wanted to gain insight into the reasons for ALS patients requesting EAS and compare these with the reasons of cancer and heart failure patients. Knowing disease-specific reasons for requesting EAS may improve palliative care in these vulnerable patients. The data used in the present study were derived from the Support and Consultation in Euthanasia in The Netherlands (SCEN) evaluation study. This study provided consultation reports and questionnaires filled out by the attending physicians from 3,337 consultations conducted by SCEN physicians in situations where a patient requested EAS. For this study we selected data on all ALS patients $(n=51)$, all heart failure patients $(n=61)$, and a random sample of 73 cancer patients. The most frequently reported reasons for unbearable suffering
\end{abstract}

Electronic supplementary material The online version of this article (doi:10.1007/s00415-010-5474-y) contains supplementary material, which is available to authorized users.

M. Maessen · J. H. Veldink ( $ه)$ - L. H. van den Berg ·

H. J. Schouten

Department of Neurology, University Medical Centre Utrecht, P.O. Box 85500, 3508 GA Utrecht, The Netherlands

e-mail: jveldink@umcutrecht.nl

M. Maessen

e-mail: m.maessen-2@umcutrecht.nl

G. van der Wal · B. D. Onwuteaka-Philipsen

Department of Public and Occupational Health, The EMGO

Institute, VU University Medical Centre, Amsterdam,

The Netherlands were: fear of suffocation (45\%) and dependency (29\%) in ALS patients, pain $(46 \%)$ and fatigue $(28 \%)$ in cancer patients, and dyspnea (52\%) and dependency (37\%) in heart failure patients. Somatic complaints were reported more frequently as a reason for EAS by cancer patients [odds ratio (OR) 0.20 , 95\% confidence interval (CI) 0.09 0.46 ] and heart failure patients [OR $0.16,95 \%$ CI $0.05-$ 0.46] than by ALS patients. ALS patients should be helped in a timely fashion to cope with psychosocial symptoms, e.g., by informing them about the low risk of suffocation in the terminal phase and the possible means of preventing this.

Keywords Amyotrophic lateral sclerosis . Cardiovascular diseases - Cancer - Palliative care . Hasten death

\section{Introduction}

In the final stage of life, patients often suffer from physical, psychosocial or existential symptoms. When this suffering becomes unbearable, patients may request the physician to hasten their death [2] or explicitly for euthanasia or physician-assisted suicide (EAS), even if it is not legal for physicians to perform this $[1,14,18]$. In most countries, it is currently illegal for physicians to grant such requests. In The Netherlands, where EAS is allowed under certain circumstances, $1.8 \%$ of all deaths in 2005 were the result of EAS [13]. Public perception is that EAS is more frequently associated with cancer, as this is far commoner. Of all cancer patients who died in The Netherlands, EAS was performed in 5\% [13], compared with $20 \%$ of ALS patient deaths [16] and $0.5 \%$ of heart failure patient deaths [15]. 
One of the mandatory conditions in Dutch law for performing EAS is that the patient requesting EAS is suffering unbearably and the outlook is hopeless. More insight into the disease-specific aspects of suffering which cause patients to prefer death over life may help to improve palliative care.

In the present study, we wanted to gain insight into the physical and psychological symptoms of ALS patients who requested EAS and into the factors that made their suffering unbearable. To study disease-specific differences, we compared the results of ALS patients with those of cancer and heart failure patients, two groups with a lower EAS proportion than in ALS patients. Together, these three groups represent a disease with a high EAS proportion (ALS), a disease with a low EAS proportion (heart failure), and a disease most frequently associated with EAS (cancer).

\section{Patients and methods}

\section{Design and population}

The data used in this study were collected for the Support and Consultation in Euthanasia in The Netherlands (SCEN) evaluation study [10]. SCEN is a governmental healthcare organization that provides physicians (SCEN physicians) who can be consulted for a second opinion by treating physicians of patients who are considering or requesting EAS. SCEN physicians can also give expert advice to colleagues who have questions about EAS. SCEN physicians are trained in palliative care and the requirements of due care for performing EAS according to Dutch law. During a formal consultation, the SCEN physician talks with the treating physician, reads the patient's medical file, and visits the patient, in order to come to a judgment on whether all requirements for due care are met. The consultant records his reasoned judgment in a consultation report. Examples of summaries of such consultation reports are presented in box 1 of the Supplementary Material. In the SCEN evaluation study, all consultations by SCEN physicians were registered. Shortly after each consultation, the SCEN physician filled out a questionnaire on the consultation and sent it together with a copy of the consultation report to the researchers. SCEN physicians also handed a questionnaire to the treating physician, who could send the questionnaire back to the researchers. All questionnaires and consultation reports were anonymous, but by using a registration number, the treating physician's questionnaire could be linked to the consultant's questionnaire and report. The Medical Ethical Committee approved the study protocol.
Study population

Since 1999, SCEN has been gradually implemented in The Netherlands, being available to $60 \%$ of all primary care physicians since the first half of 2001 , and to all primary care physicians and some specialists since the second half of 2002. Consultation reports and treating physicians' questionnaires for this study were collected between April 1, 2000 and January 1, 2003.

The 495 available SCEN physicians sent a total of 3,891 SCEN questionnaires to the researchers, and 3,337 (86\%) treating physician questionnaires were also returned. From these questionnaires we selected all questionnaires on patients with diagnosis of ALS $(n=52)$ and heart failure $(n=68)$. For 51 ALS patients and 61 heart failure patients the corresponding SCEN consultation report was also available. In addition we randomly selected 80 patients from all those with diagnosis of cancer $(n=2,842)$; in 73 of them a SCEN consultant report was available as well. Both patients whose request for EAS was granted and those whose request did not result in EAS were included in this study. As SCEN was gradually and randomly implemented in The Netherlands, a random subset of all patients requesting euthanasia in The Netherlands at that time was available to us.

In the present study, euthanasia and physician-assisted suicide (EAS) was defined as follows: physicians administer, supply or prescribe a drug with the explicit intention to end the patient's life (or to enable the patient to end his or her own life), at the explicit request of the patient.

\section{Questionnaires}

Besides questions on the consultation by the SCEN physician, which were relevant for the evaluation of SCEN, the questionnaire for the consulting treating physicians contained further questions on sex, age, diagnosis, and physical and depressive symptoms at the moment of consultation of the patient requesting EAS. Symptoms of depression were measured using a short version of a validated mood dimension scale, the Nurses' Observation Scale for Geriatric Patients (NOSGER) [17]. Treating physicians also had to state whether EAS was performed.

The written consultation report of the SCEN physician consists of a text indicating the patient's medical situation, and a reasoned answer to the question whether or not the requirements for due care are met. The way of doing this was typically to discuss each of the substantive requirements for due care, including a well-considered voluntary request from the patient, the presence of unbearable and hopeless suffering, and the absence of realistic alternatives for treatment. Two independent 
researchers assessed each consultation report. They determined which aspects of suffering were the reasons for the patient's suffering being unbearable. A comprehensive list of possible aspects of suffering was used to categorize the unbearable nature of suffering at the end of life. If the two researchers did not score the same codes for suffering, they discussed the SCEN report together to reach consensus.

Data analysis

To analyze differences in sex and age, we used the chisquare test and the $t$ test. The physical and depressive symptoms were measured using a five-point scale, on which lower values indicated fewer symptoms. When patients reported 1 or 2 on these five-point scales, symptoms were assumed to be absent. When patients reported 3, 4 or 5 on the five-point scale, symptoms were assumed to be present (Tables 1,2). Differences between ALS, cancer, and heart failure patients regarding symptoms and reasons for unbearable suffering were calculated using multivariate logistic regression analysis, which was adjusted for sex and age at time of EAS request. Aspects of unbearable suffering that were based on similar underlying conditions were combined together into more general categories and are presented in Table 4 . The provided tables have a maximum of six missing patients.

\section{Results}

Patient characteristics

Forty-nine percent of the ALS patients, 37\% of the cancer patients, and $46 \%$ of the heart failure patients were women. Average age at time of EAS request was 65 years for ALS, 66 years for cancer, and 80 years for heart failure patients. The distribution of sex did not differ significantly between the three patients groups $(p \geq 0.18)$. The heart failure patients were significantly older than the ALS $(p \leq 0.001)$ and the cancer patients $(p \leq 0.001)$.

During the SCEN evaluation study, 28 of the 51 ALS patients died due to EAS, 1 patient had his or her request for EAS denied, 2 patients changed their mind, and 5 patients died before EAS could be performed. In ten patients, the study period ended before the physician decided on the EAS request, one patient died before the physician decided on the EAS, and in four patients it was unknown what the outcome was. Forty-five of the 73 cancer patients died due to EAS, 2 patients had their request for EAS denied, 4 patients changed their mind, and 7 patients died before EAS could be performed. Forty-one of the 61 heart failure patients died due to EAS, 4 patients had their request for EAS denied, 3 patients changed their mind, and 7 patients died before EAS could be performed. All other patients died before the physician decided on the

Table 1 Symptoms of patients at time of request for euthanasia or physician-assisted suicide according to treating physician

\begin{tabular}{|c|c|c|c|c|c|c|c|}
\hline \multirow[t]{2}{*}{ Symptoms } & \multirow{2}{*}{$\begin{array}{l}\text { ALS patients, } \\
\%(n=51)\end{array}$} & \multirow{2}{*}{$\begin{array}{l}\text { Cancer patients, } \\
\%(n=73)\end{array}$} & \multirow{2}{*}{$\begin{array}{l}\text { Heart failure patients, } \\
\%(n=61)\end{array}$} & \multicolumn{2}{|c|}{ ALS-cancer } & \multicolumn{2}{|c|}{ ALS-heart failure } \\
\hline & & & & $\mathrm{OR}$ & $95 \% \mathrm{CI}$ & $\mathrm{OR}$ & $95 \% \mathrm{CI}$ \\
\hline Not active & 92 & 96 & 98 & 0.49 & ns & 0.18 & $\mathrm{~ns}$ \\
\hline Feeling sick & 92 & 97 & 98 & 0.30 & $\mathrm{~ns}$ & 0.23 & ns \\
\hline Fatigue & 80 & 96 & 97 & 0.17 & $0.04-0.66$ & 0.12 & $0.02-0.68$ \\
\hline Poor appetite & 76 & 89 & 87 & 0.38 & ns & 0.18 & $0.05-0.65$ \\
\hline Dyspnea & 57 & 47 & 85 & 1.54 & $\mathrm{~ns}$ & 0.24 & $0.08-0.72$ \\
\hline Pain & 33 & 63 & 43 & 0.27 & $0.12-0.59$ & 0.70 & $\mathrm{~ns}$ \\
\hline Frequently coughing & 28 & 30 & 30 & 0.96 & $\mathrm{~ns}$ & 0.78 & $\mathrm{~ns}$ \\
\hline Anxious & 28 & 34 & 28 & 0.62 & $\mathrm{~ns}$ & 0.57 & $\mathrm{~ns}$ \\
\hline Depressed & 26 & 27 & 31 & 0.94 & $\mathrm{~ns}$ & 0.73 & $\mathrm{~ns}$ \\
\hline Nausea & 16 & 62 & 44 & 0.11 & $0.04-0.27$ & 0.34 & $0.11-0.99$ \\
\hline Vomiting & 8 & 42 & 18 & 0.09 & $0.03-0.31$ & 0.27 & $\mathrm{~ns}$ \\
\hline Pressure sores & 8 & 13 & 12 & 0.64 & $\mathrm{~ns}$ & 1.10 & $\mathrm{~ns}$ \\
\hline Decreased consciousness & 8 & 10 & 8 & 0.85 & ns & 0.67 & $\mathrm{~ns}$ \\
\hline Confused & 6 & 13 & 8 & 0.28 & ns & 0.29 & $\mathrm{~ns}$ \\
\hline
\end{tabular}

95\% Confidence interval (95\% CI) of the odds ratio (OR) for the various symptoms for amyotrophic lateral sclerosis (ALS) patients compared with cancer patients (reference group) and ALS patients compared with heart failure patients (reference group), adjusted for sex and age

$A L S$ amyotrophic lateral sclerosis, $n s$ not significant 
Table 2 Depressive symptoms of patients at time of request for euthanasia or physician-assisted suicide according to treating physician

\begin{tabular}{|c|c|c|c|c|c|c|c|}
\hline \multirow[t]{2}{*}{ Symptoms } & \multirow{2}{*}{$\begin{array}{l}\text { ALS patients, } \\
\%(n=51)\end{array}$} & \multirow{2}{*}{$\begin{array}{l}\text { Cancer patients, } \\
\%(n=73)\end{array}$} & \multirow{2}{*}{$\begin{array}{l}\text { Heart failure patients, } \\
\%(n=61)\end{array}$} & \multicolumn{2}{|c|}{ ALS-cancer } & \multicolumn{2}{|c|}{ ALS-heart failure } \\
\hline & & & & OR & $95 \% \mathrm{CI}$ & OR & $95 \% \mathrm{CI}$ \\
\hline Feeling worthless & 34 & 30 & 41 & 1.45 & ns & 1.01 & ns \\
\hline Feeling depressed & 32 & 33 & 36 & 0.90 & ns & 0.87 & ns \\
\hline Was in a happy mood & 54 & 49 & 41 & 1.32 & ns & 1.20 & ns \\
\hline Interested in upcoming events & 53 & 43 & 35 & 1.45 & ns & 1.25 & ns \\
\hline
\end{tabular}

95\% Confidence interval (95\% CI) of the odds ratio (OR) for depressive symptoms for amyotrophic lateral sclerosis (ALS) patients compared with cancer patients (reference group) and ALS patients compared with heart failure patients (reference group), adjusted for sex and age

$A L S$ amyotrophic lateral sclerosis, $n s$ not significant

Table 3 Top five most reported uncombined reasons for unbearable suffering

\begin{tabular}{llll}
\hline & ALS patients $(n=51)$ & Cancer patients $(n=73)$ & Heart failure patients $(n=61)$ \\
\hline 1 & Fear of suffocation 45\% & Pain $46 \%$ & Dyspnea 52\% \\
2 & Dependency 29\% & Fatigue 28\% & Dependency 37\% \\
3 & Loss of dignity 20\% & Loss of dignity 24\% & Knowing that the suffering will only get worse 30\% \\
4 & Dyspnea/poor communication 16\% & Dependency 18\% & Being bedridden 25\% \\
5 & Fear of dependency 14\% & Dyspnea 17\% & Pain 23\% \\
\hline
\end{tabular}

$A L S$ amyotrophic lateral sclerosis

EAS, or the study ended before the physician decided on the EAS request. Thirteen patients had missing data.

\section{Symptoms}

According to the treating physicians, ALS patients demonstrated the following symptoms less often than cancer patients: fatigue ( $80 \%$ versus $96 \%$ ), pain (33\% versus $63 \%$ ), nausea (16\% versus $62 \%$ ), and vomiting ( $8 \%$ versus $42 \%$ ). Compared with heart failure patients, ALS patients were less often fatigued ( $80 \%$ versus $97 \%$ ), affected by poor appetite $(76 \%$ versus $87 \%)$, dyspneic $(57 \%$ versus $85 \%)$, and nauseous (16\% versus $44 \%)$ (Table 1$)$.

\section{Depressive symptoms}

As shown in Table 2, no statistically significant differences in the four NOSGER items on depressive symptoms, which might indicate possible depression, were observed in ALS patients compared with cancer patients or in ALS patients compared with heart failure patients.

\section{Unbearable suffering}

Table 3 lists the five most frequently reported reasons leading to the classification "unbearable suffering" in ALS, cancer, and heart failure patients. The reasons for unbearable suffering of the patient as recorded in the SCEN consultation reports, combined into more general categories, are presented in Table 4.

Compared with cancer and heart failure patients, ALS patients reported to the SCEN physician somatic symptoms significantly less often as being unbearable. Physicians of cancer patients indicated pain and fatigue significantly more often as being unbearable than did physicians of ALS patients. Dyspnea was more often a reason for the request for heart failure patients than for ALS patients. In contrast to physicians of cancer and heart failure patients, physicians of ALS patients did not report gastrointestinal symptoms, incontinence, pressure sores, or other somatic complaints as a reason for requesting euthanasia. In addition, physicians of ALS patients reported dependency, being limited in communication, and anxiety as reasons for unbearable suffering more often than did physicians of cancer patients.

Although the significance of difference could not be tested due to small numbers, the percentages show that immobility was an important reason for unbearable suffering for all patients. Two kinds of immobility can be distinguished here: for instance, paralyzed extremities, and being bedridden due to fatigue or dyspnea. ALS patients more frequently had paralyzed extremities as a reason for their unbearable suffering (12\% ALS, $0 \%$ cancer and heart failure patients), while heart failure patients more 
Table 4 Reasons for unbearable suffering of patients with a euthanasia or physician-assisted suicide request

\begin{tabular}{|c|c|c|c|c|c|c|c|}
\hline \multirow[t]{2}{*}{ Reasons } & \multirow{2}{*}{$\begin{array}{l}\text { ALS patients, } \\
\%(n=51)\end{array}$} & \multirow{2}{*}{$\begin{array}{l}\text { Cancer patients, } \\
\%(n=73)\end{array}$} & \multirow{2}{*}{$\begin{array}{l}\text { Heart failure patients, } \\
\%(n=61)\end{array}$} & \multicolumn{2}{|c|}{ ALS-cancer } & \multicolumn{2}{|c|}{ ALS-heart failure } \\
\hline & & & & OR & $95 \% \mathrm{CI}$ & OR & $95 \% \mathrm{CI}$ \\
\hline Psychosocial & 96 & 88 & 92 & 1.26 & ns & 1.76 & ns \\
\hline Anxious $^{\mathrm{a}}$ & 65 & 39 & 25 & 2.73 & $1.26-5.92$ & 3.95 & $1.49-10.45$ \\
\hline Fear of increase in somatic complaints & 12 & 15 & 10 & 0.63 & ns & 0.60 & ns \\
\hline Fear of coma/of vegetating & 10 & 1 & 2 & 7.07 & ns & 4.97 & ns \\
\hline Fear of dependence & 14 & 7 & 2 & 1.99 & ns & 4.16 & ns \\
\hline Fear of not being able to communicate & 10 & 3 & 0 & 3.82 & ns & - & - \\
\hline Fear of suffocation & 45 & 11 & 3 & 6.61 & $2.48-17.65$ & 14.66 & $2.86-75.12$ \\
\hline Dependency $^{\mathrm{a}}$ & 39 & 21 & 42 & 2.41 & $1.05-5.55$ & 2.66 & $\mathrm{~ns}$ \\
\hline Hopelessness $^{\mathrm{a}}$ & 27 & 22 & 33 & 1.16 & ns & 0.76 & $\mathrm{~ns}$ \\
\hline Loss of dignity ${ }^{\mathrm{a}}$ & 20 & 24 & 8 & 0.70 & ns & 2.05 & ns \\
\hline Limited communication $^{\mathrm{a}}$ & 18 & 1 & 7 & 14.90 & $1.80-123.1$ & 3.84 & ns \\
\hline Limited in leisure, work or personal care ${ }^{\mathrm{a}}$ & 14 & 17 & 23 & 0.87 & ns & 0.61 & ns \\
\hline General depressive thoughts ${ }^{\mathrm{a}}$ & 12 & 15 & 3 & 0.79 & ns & 6.06 & ns \\
\hline Other psychosocial reasons ${ }^{\mathrm{a}}$ & 10 & 7 & 8 & 1.50 & ns & 3.56 & $\mathrm{~ns}$ \\
\hline Cognitive decrease $^{\mathrm{a}}$ & 2 & 7 & 3 & 0.27 & ns & 1.08 & ns \\
\hline Feeling a burden on others ${ }^{\mathrm{a}}$ & 2 & 3 & 0 & 0.76 & $\mathrm{~ns}$ & - & - \\
\hline Symptoms of depression $^{\mathrm{a}}$ & 0 & 7 & 8 & - & - & - & \\
\hline Somatic & 51 & 83 & 85 & 0.20 & $0.09-0.46$ & 0.16 & $0.05-0.46$ \\
\hline Immobility $^{\mathrm{a}}$ & 29 & 22 & 40 & 1.45 & ns & 0.84 & ns \\
\hline Dyspnea $^{\mathrm{a}}$ & 16 & 18 & 52 & 0.70 & ns & 0.12 & $(0.04-0.41)$ \\
\hline Fatigue $^{\mathrm{a}}$ & 14 & 45 & 25 & 0.22 & $(0.08-0.59)$ & 0.44 & ns \\
\hline Pain & 12 & 46 & 23 & 0.16 & $(0.06-0.42)$ & 0.30 & ns \\
\hline Difficulty swallowing & 10 & 3 & 2 & 3.58 & ns & 6.34 & ns \\
\hline Gastrointestinal complaints $^{\mathrm{a}}$ & 0 & 18 & 12 & - & - & - & - \\
\hline Incontinence $^{\mathrm{a}}$ & 0 & 4 & 12 & - & - & - & - \\
\hline Pressure sores & 0 & 4 & 10 & - & - & - & - \\
\hline Other somatic reasons ${ }^{\mathrm{a}}$ & 0 & 8 & 18 & - & - & - & - \\
\hline
\end{tabular}

95\% Confidence interval (95\% CI) of the odds ratio (OR) for the various reasons for unbearable suffering for amyotrophic lateral sclerosis (ALS) patients compared with cancer patients (reference group) and ALS patients compared with heart failure patients (reference group), adjusted for sex and age

$A L S$ amyotrophic lateral sclerosis

-, cannot be calculated due to empty cells

a Similar reasons for unbearable suffering are combined together in this more general category

frequently had being bedridden as the reason for their unbearable suffering ( $25 \%$ heart failure patients, $4 \%$ cancer patients, 0\% ALS patients). None of the SCEN physicians of ALS patients reported symptoms of depression as a reason for unbearable suffering. Seven percent of the cancer patients and $8 \%$ of the heart failure patients scored at least one symptom of depression. These symptoms of depression consisted of three subcategories based on the DSM IV classification of depression: feeling excessively guilty, depressed mood, and diminished interest or pleasure in all, or almost all, activities for most of the day, nearly every day, for a minimum of two consecutive weeks.

\section{Discussion}

This study showed that fear of suffocation is the most frequently mentioned reason for unbearable suffering in ALS patients presenting with an EAS request. In cancer and heart failure patients with an EAS request, somatic complaints are the most important reasons for unbearable suffering.

Strengths of our study are the high response rate, the anonymity of the respondents, and minimal chance of recall bias because the reports were completed shortly after the consultation. A limitation is the lack of patient 
perspectives on symptoms and suffering, as the respondents were consultants and treating physicians. Another limitation is the fact that the treating physician reported symptoms of the patients using a standard list, which did not include all typical disease-specific symptoms. Nevertheless, in the SCEN reports, in which physicians were free to report every type of suffering, we found comparable physical symptoms. Furthermore, this study focuses mainly on primary care physicians, who in fact do receive the majority of requests for EAS in The Netherlands [11].

Previous research showed that ALS patients who died by EAS did not differ from ALS patients who did not request EAS regarding physical function (mobility and communication) [16]. This indicates that there may be factors, other than physical symptoms, that explain the high proportion of EAS in ALS patients. In our study, treating physicians of ALS patients with an EAS request reported fewer physical symptoms compared with treating physicians of cancer and heart failure patients with an EAS request. These findings suggest that, in ALS patients, psychosocial reasons and especially anxiety may play a larger role in unbearable suffering than in cancer and heart failure patients. The fewer physical symptoms in ALS patients reported by the treating physician correspond to the lower frequency of somatic complaints reported by SCEN physicians as a reason for unbearable suffering of ALS patients compared with cancer and heart failure patients.

Dependence on others is a psychosocial reason for suffering being unbearable that is mentioned frequently by all patient groups. Dependency in ALS patients often is the consequence of paralysis, while for cancer and heart failure patients, dependency usually is caused by fatigue or dyspnea.

Median survival of ALS patients after onset of first symptoms is $3-5$ years, although survival of more than 10 years has been reported [7]. The first symptoms of ALS are usually muscle weakness and increased dependency on others [12]. Psychosocial suffering in ALS therefore may not be restricted to the terminal phase. In general, muscle weakness in ALS may lead to more severe dependence for a longer period of time than in cancer and heart failure.

A possible explanation for the higher proportion of EAS in ALS patients may be the lack of palliative alternatives. Suffering caused by pain in cancer patients, or dyspnea in heart failure patients, can be relieved by opioids, and in the case of refractory symptoms by palliative sedation. In psychosocial suffering in ALS patients, these treatments are not an adequate option. In addition, for palliative sedation, guidelines from the Royal Dutch Medical Association stipulate that life expectancy in continuous deep sedation should be 2 weeks or less. Therefore it cannot be a solution for medical or psychosocial problems in ALS that occur before the terminal phase [5]. As psychosocial symptoms cannot always be prevented, patients should be helped to cope with these symptoms at the earliest possible stage [4].

Although the percentage of ALS patients that actually die following acute respiratory distress is small $(0-3 \%)$, a large percentage of ALS patients are afraid of suffocation $[3,6,9]$. A retrospective study carried out in ALS caregivers, however, reported that $52 \%$ of ALS patients had moderate to severe choking episodes during the last month of their life [8]. Adequately informing ALS patients about the limited chance of experiencing an unpleasant death due to suffocation and about ways of preventing this may diminish their fear, ease their suffering, and might influence the high EAS rate among ALS patients.

\section{Conclusions}

Somatic complaints were reported significantly more frequently as a reason for EAS by cancer and heart failure patients than by ALS patients. ALS patients reported more often fear, dependency, and communication problems; however, it cannot be concluded that the high percentage of EAS in ALS is a causal result of these symptoms. Nevertheless, these specific psychosocial symptoms are important factors that need special attention when caring for ALS patients.

\section{References}

1. Asai A, Ohnishi M, Nagata SK, Tanida N, Yamazaki Y (2001) Doctors' and nurses' attitudes towards and experiences of voluntary euthanasia: survey of members of the Japanese Association of Palliative Medicine. J Med Ethics 27:324-330

2. Borasio GD, Voltz R (1998) Discontinuation of mechanical ventilation in patients with amyotrophic lateral sclerosis. J Neurol 245:717-722

3. Bradley WG, Anderson F, Bromberg M, Gutmann L, Harati Y, Ross M, Miller RG (2001) Current management of ALS: comparison of the ALS CARE database and the AAN practice parameter. The American Academy of Neurology. Neurology 57:500-504

4. Chio A, Gauthier A, Montuschi A, Calvo A, Di VN, Ghiglione P, Mutani R (2004) A cross sectional study on determinants of quality of life in ALS. J Neurol Neurosurg Psychiatry 75:15971601

5. Committee on National Guidelines for Palliative Sedation Royal Dutch Medical Association (KNMG) (2005) Guidelines for palliative sedation of Royal Dutch Medical Association (KMMG)

6. Corcia P, Pradat PF, Salachas F, Bruneteau G, Forestier N, Seilhean D, Hauw JJ, Meininger V (2008) Causes of death in a post-mortem series of ALS patients. Amyotroph Lateral Scler 9:59-62 
7. Czaplinski A, Yen AA, Appel SH (2006) Amyotrophic lateral sclerosis: early predictors of prolonged survival. J Neurol 253:1428-1436

8. Ganzini L, Johnston WS, Silveira MJ (2002) The final month of life in patients with ALS. Neurology 59:428-431

9. Neudert C, Oliver D, Wasner M, Borasio GD (2001) The course of the terminal phase in patients with amyotrophic lateral sclerosis. J Neurol 248:612-616

10. Onwuteaka-Philipse BD, Jansen-van der Weide MC, Pasman HRW, van der Wal G (2003) Support and consultation in euthanasia in the Netherlands (in Dutch). KNMG, Amsterdam

11. Onwuteaka-Philipsen BD, van der Heide A, Koper D, Keij-Deerenberg I, Rietjens JA, Rurup ML, Vrakking AM, Georges JJ, Muller MT, van der Wal G, van der Maas PJ (2003) Euthanasia and other end-of-life decisions in the Netherlands in 1990, 1995, and 2001. Lancet 362:395-399

12. Phukan J, Hardiman O (2009) The management of amyotrophic lateral sclerosis. J Neurol 256:176-186

13. van der Heide A, Onwuteaka-Philipsen BD, Rurup ML, Buiting HM, van Delden JJ, Hanssen-de Wolf JE, Janssen AG, Pasman HR, Rietjens JA, Prins CJ, Deerenberg IM, Gevers JK, van der Maas PJ, van der Wal G (2007) End-of-life practices in the
Netherlands under the Euthanasia Act. N Engl J Med 356:19571965

14. van der Heide A, Deliens L, Faisst K, Nilstun T, Norup M, Paci E, van der WG, van der Maas PJ (2003) End-of-life decisionmaking in six European countries: descriptive study. Lancet 362:345-350

15. van der Wal G, Onwuteaka-Philipsen BD (1996) Cases of euthanasia and assisted suicide reported to the public prosecutor in North Holland over 10 years. BMJ 312:612-613

16. Veldink JH, Wokke JH, van der Wal G, Vianney de Jong JM, van den Berg LH (2002) Euthanasia and physician-assisted suicide among patients with amyotrophic lateral sclerosis in the Netherlands. N Engl J Med 346:1638-1644

17. Wahle M, Haller S, Spiegel R (1996) Validation of the NOSGER (Nurses' Observation Scale for Geriatric Patients): reliability and validity of a caregiver rating instrument. Int Psychogeriatr 8:525547

18. Wilson KG, Chochinov HM, McPherson CJ, Skirko MG, Allard P, Chary S, Gagnon PR, Macmillan K, De LM, O'Shea F, Kuhl D, Fainsinger RL, Karam AM, Clinch JJ (2007) Desire for euthanasia or physician-assisted suicide in palliative cancer care. Health Psychol 26:314-323 